\title{
Impulsive fractional differential inclusions with state-dependent delay
}

\author{
Khalida Aissani, Mouffak Benchohra*
}

\begin{abstract}
In this paper, we prove the existence of mild solution for impulsive fractional inclusions with state-dependent delay in Banach spaces. Our study is based on the nonlinear alternative of LeraySchauder type for multivalued maps due to Martelli. An example is provided to illustrate the main result.
\end{abstract}

\section{INTRODUCTION}

Fractional differential equations have been proved to be one of the most effective tools in the modeling of many phenomena in various fields of physics, mechanics, chemistry, engineering, etc. For more details, see [1, 2, 31, 34, $41,42,48]$. In order to describe various real-world problems in physical and engineering science subject to abrupt changes at certain instants during the evolution process, impulsive differential equations have been used to model the systems. The theory of impulsive differential equations is an important branch of differential equations, which has an extensive physical background $[9,12,33]$.

On the other hand, functional differential equations with state-dependent delay appear frequently in applications as model of equations and for this reason the study of this type of equations has received great attention in the last few years, see for instance $[4,10,23,24,25,26,27,28,44,47]$ and the references therein. The literature devoted to this subject is concerned fundamentally with first-order functional differential equations for which the state belongs to some finite dimensional space, see among other works, $[13,15,17,22,32,36,46]$. The problem of the existence and uniqueness of

2010 Mathematics Subject Classification. Primary: 26A33, Secondary: 34A08, 34A37, 34A60, 34H05, 34K09, 34K30.

Key words and phrases. Impulsive fractional differential inclusions, $\alpha$-resolvent family, solution operator, Caputo fractional derivative, mild solution, multivalued map, fixed point, Banach spaces.

Full paper. Received 17 November 2019, revised 4 December 2019, accepted 12 December 2019, available online 19 December 2019.

*Corresponding Author. E-mail: benchohra@yahoo.com 
solutions for fractional differential equations with delay was recently studied by Maraaba et al. in [37, 38]. In [5], the authors provide sufficient conditions for the existence of mild solutions for a class of fractional integro-differential equations with state-dependent delay, where as in $[7,40]$ we investigate the existence and controllability results for a class of impulsive fractional evolution equations with state-dependent delay.

El-Sayed and Ibrahim initiated the study of fractional differential inclusions in [19]. Recently several qualitative results for fractional differential inclusion several results were obtained in [14]. In [6] we establish sufficient conditions for the existence of mild solutions for fractional integro-differential inclusions with state-dependent delay in Banach spaces.

In this work we establish the existence of mild solutions for the class of impulsive fractional inclusions with state-dependent delay described by the form

$$
\begin{gathered}
D_{t}^{\alpha} x(t) \in A x(t)+F\left(t, x_{\rho\left(t, x_{t}\right)}, x(t)\right), t \in J_{k}=\left(t_{k}, t_{k+1}\right], k=0,1, \ldots, m, \\
\Delta x\left(t_{k}\right)=I_{k}\left(x\left(t_{k}^{-}\right)\right), \quad k=1,2, \ldots, m, \\
x(t)=\phi(t),
\end{gathered}
$$

where ${ }^{C} D_{t}^{\alpha}$ is the Caputo fractional derivative of order $0<\alpha<1, A: D(A) \subset$ $E \rightarrow E$ is the infinitesimal generator of an $\alpha$-resolvent family $\left(S_{\alpha}(t)\right)_{t \geq 0}, F: J \times \mathcal{B} \times$ $E \longrightarrow \mathcal{P}(E)$ is a multivalued map $(\mathcal{P}(E)$ is the family of all nonempty subsets of the separable Banach space $(E,\|\cdot\|)$ and $J=[0, T], T>0$, and $\phi \in \mathcal{B}$ with $\phi(0)=0$. Here, $0=t_{0}<t_{1}<\ldots<t_{m}<t_{m+1}=T, I_{k}: E \rightarrow E, k=1,2, \ldots, m$, are maps, $\Delta x\left(t_{k}\right)=x\left(t_{k}^{+}\right)-x\left(t_{k}^{-}\right), x\left(t_{k}^{+}\right)=\lim _{h \rightarrow 0} x\left(t_{k}+h\right)$ and $x\left(t_{k}^{-}\right)=\lim _{h \rightarrow 0} x\left(t_{k}-h\right)$ represent the right and the left limit of $x(t)$ at $t=t_{k}$, respectively. We denote by $x_{t}$ the element of $\mathcal{B}$ defined by $x_{t}(\theta)=x(t+\theta), \theta \in(-\infty, 0]$. Here $x_{t}$ represents the history of the state from $-\infty$ up to the present time $t$. We assume that the histories $x_{t}$ belongs to some abstract phase space $\mathcal{B}$, to be specified later, and $\phi \in \mathcal{B}$.

\section{Preliminaries}

We will briefly recall some basic definitions and facts from multivalued analysis that we will use in the sequel.

$C=C(J, E)$ denotes the Banach space of continuous functions from $J$ into $E$ with the norm

$$
\|y\|_{C}=\sup \{\|y(t)\|: t \in J\} .
$$

Let $L(E)$ be the Banach space of all linear and bounded operators on $E$. Let $L^{1}(J, E)$ be the space of $E$-valued Bochner integrable functions on $J$ with the norm

$$
\|y\|_{L^{1}}=\int_{0}^{T}\|y(t)\| d t .
$$

Denote by $P_{c l}(E)=\{Y \in P(E): Y$ closed $\}, \quad P_{b}(E)=\{Y \in P(E): Y$ bounded $\}$, $P_{c p}(E)=\{Y \in P(E): Y$ compact $\}, P_{c p, c}(E)=\{Y \in P(E): Y$ compact, convex $\}$, $P_{c l, c}(E)=\{Y \in P(E): Y$ closed, convex $\}$. 
A multivalued map $G: E \rightarrow P(E)$ is convex (closed) valued if $G(E)$ is convex (closed) for all $x \in E$. $G$ is bounded on bounded sets if $G(B)=\cup_{x \in B} G(x)$ is bounded in $E$ for all $B \in P_{b}(E)$ (i.e. $\sup _{x \in B}\{\sup \{\|y\|: y \in G(x)\}\}<\infty$ ).

$G$ is called upper semi-continuous (u.s.c.) on $E$ if for each $x_{0} \in E$ the set $G\left(x_{0}\right)$ is a nonempty, closed subset of $E$, and if for each open set $U$ of $E$ containing $G\left(x_{0}\right)$, there exists an open neighborhood $V$ of $x_{0}$ such that $G(V) \subseteq U$.

$G$ is said to be completely continuous if $G(B)$ is relatively compact for every $B \in P_{b}(E)$. If the multivalued map $G$ is completely continuous with nonempty compact values, then $G$ is u.s.c. if and only if $G$ has a closed graph (i.e. $x_{n} \longrightarrow$ $x_{*}, y_{n} \longrightarrow y_{*}, y_{n} \in G\left(x_{n}\right)$ imply $\left.y_{*} \in G\left(x_{*}\right)\right)$. For more details on multivalued maps see the books of Deimling [18], Górniewicz [20] and $\mathrm{Hu}$ and Papageorgiou $[30]$.

Definition 2.1. The multivalued map $F: J \times \mathcal{B} \times E \longrightarrow \mathcal{P}(E)$ is said to be Carathéodory if

(i) $t \longmapsto F(t, x, y)$ is measurable for each $(x, y) \in \mathcal{B} \times E$;

(ii) $(x, y) \longmapsto F(t, x, y)$ is upper semicontinuous for almost all $t \in J$.

Definition 2.2. Let $\alpha>0$ and $f \in L^{1}(J, E)$. The Riemann-Liouville integral is defined by

$$
I_{t}^{\alpha} f(t)=\frac{1}{\Gamma(\alpha)} \int_{0}^{t} \frac{f(s)}{(t-s)^{1-\alpha}} d s .
$$

For more details on the Riemann-Liouville fractional derivative, we refer the reader to [16].

Definition 2.3. [42]. The Caputo derivative of order $\alpha$ for a function $f: J \rightarrow E$ is defined by

$$
D_{t}^{\alpha} f(t)=\frac{1}{\Gamma(n-\alpha)} \int_{0}^{t} \frac{f^{(n)}(s)}{(t-s)^{\alpha+1-n}} d s=I^{n-\alpha} f^{(n)}(t), \quad t>0, n-1 \leq \alpha<n .
$$

If $0 \leq \alpha<1$, then

$$
D_{t}^{\alpha} f(t)=\frac{1}{\Gamma(1-\alpha)} \int_{0}^{t} \frac{f^{\prime}(s)}{(t-s)^{\alpha}} d s .
$$

Obviously, The Caputo derivative of a constant is equal to zero.

In order to defined the mild solution of the problems (1) we recall the following definition.

Definition 2.4. A closed and linear operator $A$ is said to be sectorial if there are constants $\omega \in \mathbb{R}, \theta \in\left[\frac{\pi}{2}, \pi\right], M>0$, such that the following two conditions are satisfied:

(1) $\sum_{(\theta, \omega)}:=\{\lambda \in C: \lambda \neq \omega,|\arg (\lambda-\omega)|<\theta\} \subset \rho(A)(\rho(A)$ being the resolvent set of $A)$.

(2) $\|R(\lambda, A)\|_{L(E)} \leq \frac{M}{|\lambda-\omega|}, \quad \lambda \in \sum_{(\theta, \omega)}$.

Sectorial operators are well studied in the literature. For details see [24].

Definition 2.5. [8] if $A$ is a closed linear operator with domain $D(A)$ defined on a Banach space $E$ and $\alpha>0$, then we say that $A$ is the generator of an $\alpha$-resolvent 
family if there exists $\omega \geq 0$ and a strongly continuous function $S_{\alpha}: \mathbb{R}_{+} \rightarrow L(E)$ such that $\left.\left\{\lambda^{\alpha}: \operatorname{Re}(\lambda)>\omega\right\} \subset \rho(A)\right)$ and

$$
\left(\lambda^{\alpha} I-A\right)^{-1} x=\int_{0}^{\infty} e^{-\lambda t} S_{\alpha}(t) x d t, \quad \operatorname{Re} \lambda>\omega, x \in E .
$$

In this case, $S_{\alpha}(t)$ is called the $\alpha$-resolvent family generated by $A$.

Definition 2.6. (see Definition 2.1 in [3]) if $A$ is a closed linear operator with domain $D(A)$ defined on a Banach space $E$ and $\alpha>0$, then we say that $A$ is the generator of a solution operator if there exist $\omega \geq 0$ and a strongly continuous function $S_{\alpha}: \mathbb{R}_{+} \rightarrow L(E)$ such that $\left\{\lambda^{\alpha}: \operatorname{Re}(\lambda)>\omega\right\} \subset \rho(A)$ and

$$
\lambda^{\alpha-1}\left(\lambda^{\alpha} I-A\right)^{-1} x=\int_{0}^{\infty} e^{-\lambda t} S_{\alpha}(t) x d t, \quad \operatorname{Re} \lambda>\omega, x \in E,
$$

in this case, $S_{\alpha}(t)$ is called the solution operator generated by $A$. For more details see $[36,43]$.

In this paper, we will employ an axiomatic definition for the phase space $\mathcal{B}$ which is similar to those introduced by Hale and Kato [21]. Specifically, $\mathcal{B}$ will be a linear space of functions mapping $(-\infty, 0]$ into $E$ endowed with a seminorm $\|\cdot\|_{\mathcal{B}}$, and satisfies the following axioms:

(A1): If $x:(-\infty, T] \longrightarrow E$ is such that $x_{0} \in \mathcal{B}$, then for every $t \in J$, $x_{t} \in \mathcal{B}$ and

$$
\|x(t)\| \leq C\left\|x_{t}\right\|_{\mathcal{B}},
$$

where $C>0$ is a constant.

(A2): There exist a continuous function $C_{1}(t)>0$ and a locally bounded function $C_{2}(t) \geq 0$ in $t \geq 0$ such that

$$
\left\|x_{t}\right\|_{\mathcal{B}} \leq C_{1}(t) \sup _{s \in[0, t]}\|x(s)\|+C_{2}(t)\left\|x_{0}\right\|_{\mathcal{B}}
$$

for $t \in[0, T]$ and $x$ as in $(A 1)$.

(A3): The space $\mathcal{B}$ is complete.

\section{Example 2.1. The phase space $C_{r} \times L^{p}(g, X)$.}

Let $r \geq 0,1 \leq p<\infty$, and let $g:(-\infty,-r) \rightarrow \mathbb{R}$ be a nonnegative measurable function which satisfies the conditions $(g-5),(g-6)$ in the terminology of [29]. Briefly, this means that $g$ is locally integrable and there exists a nonnegative, locally bounded function $\Lambda$ on $(-\infty, 0]$, such that $g(\xi+\theta) \leq \Lambda(\xi) g(\theta)$, for all $\xi \leq 0$ and $\theta \in(-\infty,-r) \backslash N_{\xi}$, where $N_{\xi} \subseteq(-\infty,-r)$ is a set with Lebesgue measure zero.

The space $C_{r} \times L^{p}(g, X)$ consists of all classes of functions $\varphi:(-\infty, 0] \rightarrow X$, such that $\varphi$ is continuous on $[-r, 0]$, Lebesgue-measurable, and $g\|\varphi\|^{p}$ on $(-\infty,-r)$. The seminorm in $\|.\|_{\mathcal{B}}$ is defined by

$$
\|\varphi\|_{\mathcal{B}}=\sup _{\theta \in[-r, 0]}\|\varphi(\theta)\|+\left(\int_{-\infty}^{-r} g(\theta)\|\varphi(\theta)\|^{p} d \theta\right)^{\frac{1}{p}} .
$$

The space $\mathcal{B}=C_{r} \times L^{p}(g, X)$ satisfies axioms (A1), (A2), (A3). Moreover, for $r=0$ and $p=2$, this space coincides with $C_{0} \times L^{2}(g, X), H=1, M(t)=$ $\Lambda(-t)^{\frac{1}{2}}, K(t)=1+\left(\int_{-r}^{0} g(\tau) d \tau\right)^{\frac{1}{2}}$, for $t \geq 0$ (see [29], Theorem 1.3.8 for details). 
Let $S_{F \circ x}$ be a set defined by

$$
S_{F, x}=\left\{v \in L^{1}(J, E): v(t) \in F\left(t, x_{\rho\left(t, x_{t}\right)}, x(t)\right) \text { a.e. } t \in J\right\} .
$$

Lemma 2.1. [35]. Let $F: J \times \mathcal{B} \times E \longrightarrow P_{c p, c}(E)$ be an $L^{1}$-Carathéodory multivalued map and let $\Psi$ be a linear continuous mapping from $L^{1}(J, E)$ to $C(J, E)$, then the operator

$$
\begin{aligned}
\Psi \circ S_{F}: C(J, E) & \longrightarrow P_{c p, c}(C(J, E)), \\
x & \longmapsto\left(\Psi \circ S_{F}\right)(x):=\Psi\left(S_{F \circ x}\right)
\end{aligned}
$$

is a closed graph operator in $C(J, E) \times C(J, E)$.

The following is the multivalued version of the fixed-point theorem due to Martelli [39].

Lemma 2.2. Let $X$ be a Banach space, and $N: X \rightarrow P_{c p, c v}(X)$ be an upper semicontinuous and completely continuous multivalued map. If the set

$$
D=\{u \in X: \lambda u \in N u \text { for some } \lambda>1\}
$$

is bounded, then $N$ has a fixed point.

\section{MAin RESUlts}

Before going further we need the following lemma ([45]).

Lemma 3.1. Consider the Cauchy problem

$$
\begin{aligned}
D_{t}^{\alpha} x(t) & =A x(t)+F(t), \quad 0<\alpha<1, \\
x(0) & =x_{0},
\end{aligned}
$$

if $F$ satisfies the uniform Holder condition with exponent $\beta \in(0,1]$ and $A$ is a sectorial operator, then the unique solution of the Cauchy problem (2) is given by

$$
x(t)=T_{\alpha}(t) x_{0}+\int_{0}^{t} S_{\alpha}(t-s) F(s) d s,
$$

where

$$
\begin{aligned}
T_{\alpha}(t) & =\frac{1}{2 \pi i} \int_{\hat{B_{r}}} e^{\lambda t} \frac{\lambda^{\alpha-1}}{\lambda^{\alpha}-A} d \lambda, \\
S_{\alpha}(t) & =\frac{1}{2 \pi i} \int_{\hat{B}_{r}} e^{\lambda t} \frac{1}{\lambda^{\alpha}-A} d \lambda,
\end{aligned}
$$

$\hat{B}_{r}$ denotes the Bromwich path. $S_{\alpha}(t)$ is called the $\alpha$-resolvent family and $T_{\alpha}(t)$ is the solution operator, generated by $A$.

Theorem 3.1. [11,45] If $\alpha \in(0,1)$ and $A \in \mathbb{A}^{\alpha}\left(\theta_{0}, \omega_{0}\right)$, then for any $x \in E$ and $t>0$, we have

$$
\left\|T_{\alpha}(t)\right\|_{L(E)} \leq M e^{\omega t} \text { and }\left\|S_{\alpha}(t)\right\|_{L(E)} \leq C e^{\omega t}\left(1+t^{\alpha-1}\right), t>0, \omega>\omega_{0} .
$$

Let

$$
\widetilde{M}_{T}=\sup _{0 \leq t \leq T}\left\|T_{\alpha}(t)\right\|_{L(E)}, \quad \widetilde{M}_{s}=\sup _{0 \leq t \leq T} C e^{\omega t}\left(1+t^{\alpha-1}\right),
$$

so we have

$$
\left\|T_{\alpha}(t)\right\|_{L(E)} \leq \widetilde{M}_{T},\left\|S_{\alpha}(t)\right\|_{L(E)} \leq t^{\alpha-1} \widetilde{M}_{s}
$$


Let us consider the set of functions

$$
\begin{gathered}
\mathcal{B}_{1}=\left\{x:(-\infty, T] \rightarrow E \text { such that }\left.x\right|_{J} \in C(J, E)\right. \text { and there exist } \\
\left.x\left(t_{k}^{+}\right) \text {and } x\left(t_{k}^{-}\right) \text {with } x\left(t_{k}\right)=x\left(t_{k}^{-}\right), x_{0}=\phi, k=1,2, \ldots, m\right\},
\end{gathered}
$$

where $\left.x\right|_{J}$ is the restriction of $x$ to $J_{k}=\left(t_{k}, t_{k+1}\right], k=1,2, \ldots, m$.

From Lemma 3.1, we can define the mild solution of system (1) as follows:

Definition 3.1. A function $x:(-\infty, T] \rightarrow E$ is called a mild solution of (1) if the following holds: $x_{0}=\phi \in \mathcal{B}$ on $(-\infty, 0]$ with $\phi(0)=0,\left.\Delta x\right|_{t=t_{k}}=I_{k}\left(x\left(t_{k}^{-}\right)\right), k=$ $1,2, \ldots, m$, the restriction of $x(\cdot)$ to the interval $[0, T]$ is continuous and there exists $v(\cdot) \in L^{1}\left(J_{k}, E\right)$, such that $v(t) \in F\left(t, x_{t}, x(t)\right)$ a.e. $t \in[0, T]$, and $x$ satisfies the following integral equation:

$$
x(t)= \begin{cases}\phi(t), & t \in(-\infty, 0] ; \\ \int_{0}^{t} S_{\alpha}(t-s) v(s) d s, & t \in\left[0, t_{1}\right] ; \\ T_{\alpha}\left(t-t_{1}\right)\left(x\left(t_{1}^{-}\right)+I_{1}\left(x\left(t_{1}^{-}\right)\right)\right) & \\ +\int_{t_{1}}^{t} S_{\alpha}(t-s) v(s) d s, & t \in\left(t_{1}, t_{2}\right] ; \\ \quad \vdots & \\ T_{\alpha}\left(t-t_{m}\right)\left(x\left(t_{m}^{-}\right)+I_{m}\left(x\left(t_{m}^{-}\right)\right)\right) & \\ +\int_{t_{m}}^{t} S_{\alpha}(t-s) v(s) d s, & t \in\left(t_{m}, T\right] .\end{cases}
$$

Set

$$
\mathcal{R}\left(\rho^{-}\right)=\{\rho(s, \varphi):(s, \varphi) \in J \times \mathcal{B}, \rho(s, \varphi) \leq 0\} .
$$

We always assume that $\rho: J \times \mathcal{B} \rightarrow(-\infty, T]$ is continuous. Additionally, we introduce following hypothesis:

$\left(H_{\varphi}\right)$ The function $t \rightarrow \varphi_{t}$ is continuous from $\mathcal{R}\left(\rho^{-}\right)$into $\mathcal{B}$ and there exists a continuous and bounded function $L^{\phi}: \mathcal{R}\left(\rho^{-}\right) \rightarrow(0, \infty)$ such that

$$
\left\|\phi_{t}\right\|_{\mathcal{B}} \leq L^{\phi}(t)\|\phi\|_{\mathcal{B}} \quad \text { for every } t \in \mathcal{R}\left(\rho^{-}\right) \text {. }
$$

Remark 3.1. The condition $\left(H_{\varphi}\right)$, is frequently verified by continuous and bounded functions. For more details, see for instance [29].

Remark 3.2. In the rest of this section, $C_{1}^{*}$ and $C_{2}^{*}$ are the constants

$$
C_{1}^{*}=\sup _{s \in J} C_{1}(s) \text { and } C_{2}^{*}=\sup _{s \in J} C_{2}(s) .
$$

Lemma 3.2. [28] If $x:(-\infty, T] \rightarrow X$ is a function such that $x_{0}=\phi$, then

$$
\left\|x_{s}\right\|_{\mathcal{B}} \leq\left(C_{2}^{*}+L^{\phi}\right)\|\phi\|_{\mathcal{B}}+C_{1}^{*} \sup \{|y(\theta)| ; \theta \in[0, \max \{0, s\}]\}, s \in \mathcal{R}\left(\rho^{-}\right) \cup J,
$$

where $L^{\phi}=\sup _{t \in \mathcal{R}\left(\rho^{-}\right)} L^{\phi}(t)$.

Let us introduce the following hypotheses:

(H1) The semigroup $S(t)$ is compact for $t>0$.

(H2) The multivalued map $F: J \times \mathcal{B} \times E \longrightarrow \mathcal{P}(E)$ is Carathéodory with compact convex values.

(H3) There exist a function $\mu \in L^{1}\left(J, \mathbb{R}^{+}\right)$and a continuous nondecreasing function $\psi: \mathbb{R}^{+} \rightarrow(0,+\infty)$ such that

$$
\|F(t, x, y)\| \leq \mu(t) \psi\left(\|x\|_{\mathcal{B}}+\|y\|_{E}\right), \quad(t, x, y) \in J \times \mathcal{B} \times E,
$$


with

$$
\omega_{2} \int_{0}^{T} \mu(s) d s<\int_{v(0)}^{+\infty} \frac{d u}{\psi(u)},
$$

where

$$
\begin{gathered}
\omega_{2}=\frac{\beta_{2} \widetilde{M}_{s} T^{\alpha}}{\alpha\left(1-\widetilde{M}_{T}\right)}, \\
v(0)=\omega_{1}=\beta_{1}+\frac{\beta_{2} \widetilde{M}_{T} \Omega}{1-\widetilde{M}_{T}},
\end{gathered}
$$

and

$$
\beta_{1}=\left(C_{2}^{*}+L^{\phi}\right)\|\phi\|_{\mathcal{B}}, \beta_{2}=C_{1}^{*}+1 .
$$

(H4) The functions $I_{k}: E \rightarrow E$ are continuous, and send bounded sets into bounded sets.

Set

$$
\Omega=\max _{1 \leq k \leq m} \sup \left\{\left\|I_{k}(x)\right\|, x \in B\right\},
$$

where $B$ is a bounded set of $E$.

Theorem 3.2. Assume that assumptions $\left(H_{\varphi}\right),(H 1)-(H 4)$ hold. If $\widetilde{M_{T}}<1$, then the IVP (1) has a mild solution on $(-\infty, T]$.

Proof. We transform the problem (1) into a fixed-point problem. Consider the multivalued operator $N: \mathcal{B}_{1} \longrightarrow \mathcal{P}\left(\mathcal{B}_{1}\right)$ defined by $N(h)=\left\{h \in \mathcal{B}_{1}\right\}$ with

$$
h(t)= \begin{cases}\phi(t), & t \in(-\infty, 0] \\ \int_{0}^{t} S_{\alpha}(t-s) v(s) d s, & t \in\left[0, t_{1}\right] \\ T_{\alpha}\left(t-t_{1}\right)\left(x\left(t_{1}^{-}\right)+I_{1}\left(x\left(t_{1}^{-}\right)\right)\right)+\int_{t_{1}}^{t} S_{\alpha}(t-s) v(s) d s, & t \in\left(t_{1}, t_{2}\right] \\ \quad \vdots & \\ T_{\alpha}\left(t-t_{m}\right)\left(x\left(t_{m}^{-}\right)+I_{m}\left(x\left(t_{m}^{-}\right)\right)\right)+\int_{t_{m}}^{t} S_{\alpha}(t-s) v(s) d s, & t \in\left(t_{m}, T\right] .\end{cases}
$$

It is clear that the fixed points of the operator $N$ are mild solutions of the problem $(1)$. Let us define $y(\cdot):(-\infty, T] \longrightarrow E$ as

$$
y(t)= \begin{cases}\phi(t), & t \in(-\infty, 0] \\ 0, & t \in J\end{cases}
$$

Then $y_{0}=\phi$. For each $z \in C(J, E)$ with $z(0)=0$, we denote by $\bar{z}$ the function defined by

$$
\bar{z}(t)= \begin{cases}0, & t \in(-\infty, 0] ; \\ z(t), & t \in J\end{cases}
$$


Let $x_{t}=y_{t}+\bar{z}_{t}, t \in J$. It is easy to see that $x(\cdot)$ satisfies (3) if and only if $z_{0}=0$ and for $t \in J$, we have

$$
z(t)= \begin{cases}\int_{0}^{t} S_{\alpha}(t-s) v(s) d s, & t \in\left[0, t_{1}\right] \\ T_{\alpha}\left(t-t_{1}\right)\left[y\left(t_{1}^{-}\right)+\bar{z}\left(t_{1}^{-}\right)+I_{1}\left(y\left(t_{1}^{-}\right)+\bar{z}\left(t_{1}^{-}\right)\right)\right] & \\ +\int_{t_{1}}^{t} S_{\alpha}(t-s) v(s) d s, & t \in\left(t_{1}, t_{2}\right] \\ \quad \vdots & \\ T_{\alpha}\left(t-t_{m}\right)\left[y\left(t_{m}^{-}\right)+\bar{z}\left(t_{m}^{-}\right)+I_{m}\left(y\left(t_{m}^{-}\right)+\bar{z}\left(t_{m}^{-}\right)\right)\right] & \\ +\int_{t_{m}}^{t} S_{\alpha}(t-s) v(s) d s, & t \in\left(t_{m}, T\right],\end{cases}
$$

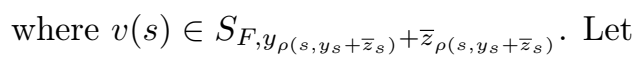

$$
\mathcal{B}_{2}=\left\{z \in \mathcal{B}_{1} \quad \text { such that } z_{0}=0\right\} .
$$

For any $z \in \mathcal{B}_{2}$, we have

$$
\begin{aligned}
\|z\|_{\mathcal{B}_{2}} & =\sup _{t \in J}\|z(t)\|+\left\|z_{0}\right\|_{\mathcal{B}} \\
& =\sup _{t \in J}\|z(t)\| .
\end{aligned}
$$

Thus $\left(\mathcal{B}_{2},\|\cdot\|_{\mathcal{B}_{2}}\right)$ is a Banach space. We define the operator $P: \mathcal{B}_{2} \longrightarrow \mathcal{P}\left(\mathcal{B}_{2}\right)$ by: $P(z)=\left\{h \in \mathcal{B}_{2}\right\}$ with

$$
h(t)= \begin{cases}\int_{0}^{t} S_{\alpha}(t-s) v(s) d s, & t \in\left[0, t_{1}\right] \\ T_{\alpha}\left(t-t_{1}\right)\left[y\left(t_{1}^{-}\right)+\bar{z}\left(t_{1}^{-}\right)+I_{1}\left(y\left(t_{1}^{-}\right)+\bar{z}\left(t_{1}^{-}\right)\right)\right] & \\ +\int_{t_{1}}^{t} S_{\alpha}(t-s) v(s) d s, & t \in\left(t_{1}, t_{2}\right] ; \\ \vdots & \\ T_{\alpha}\left(t-t_{m}\right)\left[y\left(t_{m}^{-}\right)+\bar{z}\left(t_{m}^{-}\right)+I_{m}\left(y\left(t_{m}^{-}\right)+\bar{z}\left(t_{m}^{-}\right)\right)\right] & \\ +\int_{t_{m}}^{t} S_{\alpha}(t-s) v(s) d s, & t \in\left(t_{m}, T\right],\end{cases}
$$

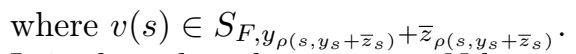

It is clear that the operator $N$ has a fixed point if and only if $P$ has a fixed point. So let us prove that $P$ has a fixed point. We shall show that the operators $P$ satisfy all conditions of Lemma 2.2. For better readability, we break the proof into a sequence of steps.

Step 1: $P$ is convex for each $z \in \mathcal{B}_{2}$.

Indeed, if $h_{1}$ and $h_{2}$ belong to $P(z)$, then there exist $v_{1}, v_{2} \in S_{F, y+\bar{z}}$ such that, for $t \in J$, we have

$$
h_{i}(t)= \begin{cases}\int_{0}^{t} S_{\alpha}(t-s) v_{i}(s) d s, & t \in\left[0, t_{1}\right] \\ T_{\alpha}\left(t-t_{1}\right)\left[y\left(t_{1}^{-}\right)+\bar{z}\left(t_{1}^{-}\right)+I_{1}\left(y\left(t_{1}^{-}\right)+\bar{z}\left(t_{1}^{-}\right)\right)\right] & \\ +\int_{t_{1}}^{t} S_{\alpha}(t-s) v_{i}(s) d s, & t \in\left(t_{1}, t_{2}\right] ; \quad(i=1,2) \\ \quad \vdots & \\ T_{\alpha}\left(t-t_{m}\right)\left[y\left(t_{m}^{-}\right)+\bar{z}\left(t_{m}^{-}\right)+I_{m}\left(y\left(t_{m}^{-}\right)+\bar{z}\left(t_{m}^{-}\right)\right)\right] & \\ +\int_{t_{m}}^{t} S_{\alpha}(t-s) v_{i}(s) d s, & t \in\left(t_{m}, T\right] .\end{cases}
$$


Let $d \in[0,1]$. Then for each $t \in\left[0, t_{1}\right]$, we get

$$
d h_{1}(t)+(1-d) h_{2}(t)=\int_{0}^{t} S_{\alpha}(t-s)\left[d v_{1}(s)+(1-d) v_{2}(s)\right] d s .
$$

Similarly, for any $t \in\left(t_{i}, t_{i+1}\right], i=1, \ldots, m$, we have

$$
\begin{aligned}
d h_{1}(t)+(1-d) h_{2}(t) & =T_{\alpha}\left(t-t_{i}\right)\left[y\left(t_{i}^{-}\right)+\bar{z}\left(t_{i}^{-}\right)+I_{i}\left(y\left(t_{i}^{-}\right)+\bar{z}\left(t_{i}^{-}\right)\right)\right] \\
& +\int_{t_{i}}^{t} S_{\alpha}(t-s)\left[d v_{1}(s)+(1-d) v_{2}(s)\right] d s
\end{aligned}
$$

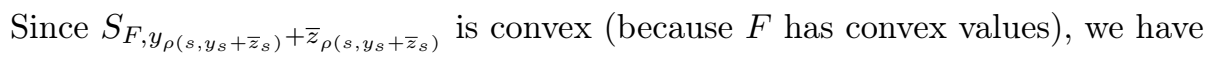

$$
d h_{1}+(1-d) h_{2} \in P(z) .
$$

Step 2: $P$ maps bounded sets into bounded sets in $\mathcal{B}_{2}$.

Indeed, it is enough to show that for any $r>0$, there exists a positive constant $\ell$ such that for each $z \in B_{r}=\left\{z \in \mathcal{B}_{2}:\|z\|_{\mathcal{B}_{2}} \leq r\right\}$, we have $\|P(z)\|_{\mathcal{B}_{2}} \leq \ell$. Let $h \in P(z)$, and using (H2) we have for each $t \in\left[0, t_{1}\right]$,

$$
\begin{aligned}
\|h(t)\| & \leq \int_{0}^{t}\left\|S_{\alpha}(t-s)\right\|\|v(s)\| d s \\
& \leq \widetilde{M}_{S} \int_{0}^{t}(t-s)^{\alpha-1} \mu(\tau) \psi\left(\left\|y_{\rho\left(s, y_{s}+\bar{z}_{s}\right)}+\bar{z}_{\rho\left(s, y_{s}+\bar{z}_{s}\right)}\right\|+\|y(s)+\bar{z}(s)\|\right) d s \\
& \leq \widetilde{M}_{S} \frac{T^{\alpha}}{\alpha} \psi\left(\left(C_{2}^{*}+L^{\phi}\right)\|\phi\|_{\mathcal{B}}+\left(C_{1}^{*}+1\right) r\right) \int_{0}^{t} \mu(s) d s \\
& \leq \widetilde{M}_{S} \frac{T^{\alpha}}{\alpha} \psi\left(\left(C_{2}^{*}+L^{\phi}\right)\|\phi\|_{\mathcal{B}}+\left(C_{1}^{*}+1\right) r\right)\|\mu\|_{L^{1}} .
\end{aligned}
$$

Moreover, when $t \in\left(t_{i}, t_{i+1}\right], i=1, \ldots, m$, we have the estimate

$$
\begin{aligned}
\|h(t)\| & \leq\left\|T_{\alpha}\left(t-t_{i}\right)\left[z\left(t_{i}^{-}\right)+I_{i}\left(z\left(t_{i}^{-}\right)\right)\right]\right\|+\int_{t_{i}}^{t}\left\|S_{\alpha}(t-s)\right\|\|v(s)\| d s \\
& \leq \widetilde{M}_{T}(r+\Omega) \\
& +\widetilde{M}_{S} \int_{t_{i}}^{t}(t-s)^{\alpha-1} \mu(\tau) \psi\left(\left\|y_{\rho\left(s, y_{s}+\bar{z}_{s}\right)}+\bar{z}_{\rho\left(s, y_{s}+\bar{z}_{s}\right)}\right\|+\|y(s)+\bar{z}(s)\|\right) d s \\
& \left.\leq \widetilde{M}_{T}(r+\Omega)+\widetilde{M}_{S} \frac{T^{\alpha}}{\alpha} \psi\left(\left(C_{2}^{*}+L^{\phi}\right) L^{\phi}\right)\|\phi\|_{\mathcal{B}}+\left(C_{1}^{*}+1\right) r\right)\|\mu\|_{L^{1}} \\
& \leq \ell .
\end{aligned}
$$

Hence $P\left(B_{r}\right)$ is bounded.

Step 3: $P$ maps bounded sets into equicontinuous sets of $\mathcal{B}_{2}$.

Let $\tau_{1}, \tau_{2} \in\left[0, t_{1}\right]$, with $\tau_{1}<\tau_{2}$, we have

$$
\begin{aligned}
\left\|h\left(\tau_{2}\right)-h\left(\tau_{1}\right)\right\| & \leq \int_{0}^{\tau_{1}}\left\|S_{\alpha}\left(\tau_{2}-s\right)-S_{\alpha}\left(\tau_{1}-s\right)\right\|\|v(s)\| d s \\
& +\int_{\tau_{1}}^{\tau_{2}}\left\|S_{\alpha}\left(\tau_{2}-s\right)\right\|\|v(s)\| d s \\
& \leq Q_{1}+Q_{2},
\end{aligned}
$$


where

$$
\begin{aligned}
Q_{1} & =\int_{0}^{\tau_{1}}\left\|S_{\alpha}\left(\tau_{2}-s\right)-S_{\alpha}\left(\tau_{1}-s\right)\right\|\|v(s)\| d s \\
& \leq \psi\left(\left(C_{2}^{*}+L^{\phi}\right)\|\phi\|_{\mathcal{B}}+\left(C_{1}^{*}+1\right) r\right)\|\mu\|_{L^{1}} \int_{0}^{\tau_{1}}\left\|S_{\alpha}\left(\tau_{2}-s\right)-S_{\alpha}\left(\tau_{1}-s\right)\right\| d s .
\end{aligned}
$$

Since $\left\|S_{\alpha}\left(\tau_{2}-s\right)-S_{\alpha}\left(\tau_{1}-s\right)\right\|_{L(E)} \leq 2 \widetilde{M}_{s}\left(t_{1}-s\right)^{\alpha-1} \in L^{1}\left(J, \mathbb{R}_{+}\right)$for $s \in\left[0, t_{1}\right]$ and $\left\|S_{\alpha}\left(\tau_{2}-s\right)-S_{\alpha}\left(\tau_{1}-s\right)\right\| \rightarrow 0$ as $\tau_{1} \rightarrow \tau_{2}$. This implies that

$$
\begin{gathered}
\lim _{\tau_{1} \rightarrow \tau_{2}} Q_{1}=0 . \\
Q_{2}=\int_{\tau_{1}}^{\tau_{2}}\left\|S_{\alpha}\left(\tau_{2}-s\right)\right\|\|v(s)\| d s \\
\leq \frac{\widetilde{M}_{s}\left(\tau_{2}-\tau_{1}\right)^{\alpha}}{\alpha} \psi\left(\left(C_{2}^{*}+L^{\phi}\right)\|\phi\|_{\mathcal{B}}+\left(C_{1}^{*}+1\right) r\right)\|\mu\|_{L^{1}} .
\end{gathered}
$$

Hence, we deduce that

$$
\lim _{\tau_{1} \rightarrow \tau_{2}} Q_{2}=0
$$

Similarly, for $\tau_{1}, \tau_{2} \in\left(t_{i}, t_{i+1}\right], i=1, \ldots, m$, we have

$$
\begin{aligned}
\left\|h\left(\tau_{2}\right)-h\left(\tau_{1}\right)\right\| & \leq\left\|T_{\alpha}\left(\tau_{2}-t_{i}\right)-T_{\alpha}\left(\tau_{1}-t_{i}\right)\right\|\left[\left\|z\left(t_{i}^{-}\right)\right\|+\left\|I_{i}\left(z\left(t_{i}^{-}\right)\right)\right\|\right]+Q_{1}+Q_{2} \\
& \leq\left\|T_{\alpha}\left(\tau_{2}-t_{i}\right)-T_{\alpha}\left(\tau_{1}-t_{i}\right)\right\|(r+\Omega)+Q_{1}+Q_{2} .
\end{aligned}
$$

Since $T_{\alpha}(t)$ is also strongly continuous, so $\left\|T_{\alpha}\left(\tau_{2}-t_{i}\right)-T_{\alpha}\left(\tau_{1}-t_{i}\right)\right\| \rightarrow 0$ as $\tau_{1} \rightarrow \tau_{2}$. Thus, from the above inequalities, we have

$$
\lim _{\tau_{1} \rightarrow \tau_{2}}\left\|h\left(\tau_{2}\right)-h\left(\tau_{1}\right)\right\|=0 .
$$

So, $P\left(B_{r}\right)$ is equicontinuous.

Step 4: The set $\left(P B_{r}\right)(t)$ is relatively compact for each $t \in J$, where

$$
\left(P B_{r}\right)(t)=\left\{h(t): h \in P\left(B_{r}\right)\right\} .
$$

For all $t \in\left[0, t_{1}\right]$, by the strong continuity of $S_{\alpha}(\cdot)$ and conditions $(H 1),(H 2)$, that the set $\left\{S_{\alpha}(t-s) v(s), t, s \in\left[0, t_{1}\right]\right\}$ is relatively compact in $E$. Moreover, from the mean value theorem for the Bochner integral, we obtain

$$
\left(P B_{r}\right)(t) \in t \overline{\operatorname{conv}}\left\{S_{\alpha}(t-s) v(s): s \in\left[0, t_{1}\right]\right\} .
$$

On the other hand, for $t \in\left(t_{i}, t_{i+1}\right], i=1, \ldots, m$, using the continuity of the operator $T_{\alpha}(\cdot)$, it follows that $\left(P B_{r}\right)(t)$ is relatively compact in $E$, for every $t \in[0, T]$. As a consequence of Step 2 to 3 together with Arzelá-Ascoli theorem we can conclude that $P$ is completely continuous.

Step 5: $P$ has a closed graph.

Suppose that $z_{n} \rightarrow z_{*}, h_{n} \in P\left(z_{n}\right)$ with $h_{n} \rightarrow h_{*}$. We claim that $h_{*} \in P\left(z_{*}\right)$.

In fact, the assumption $h_{n} \in P\left(z_{n}\right)$ implies that there exists $v_{n} \in S_{F, y_{n} \rho\left(s, y_{n s}+\bar{z}_{n s}\right)+\bar{z}_{n} \rho\left(s, y_{n s}+\bar{z}_{n s}\right)}$ such that, for each $t \in\left[0, t_{1}\right]$,

$$
h_{n}(t)=\int_{0}^{t} S_{\alpha}(t-s) v_{n}(s) d s .
$$


We will show that there exists $v_{*} \in S_{F, y_{*} \rho\left(s, y_{* s}+\bar{z}_{* s}\right)+\bar{z}_{*} \rho\left(s, y_{* s}+\bar{z}_{* s}\right)}$ such that, for each $t \in\left[0, t_{1}\right]$,

$$
h_{*}(t)=\int_{0}^{t} S_{\alpha}(t-s) v_{*}(s) d s .
$$

Consider the following linear continuous operator:

$$
\begin{aligned}
\Upsilon: L^{1}\left(\left[0, t_{1}\right], E\right) & \longrightarrow C\left(\left[0, t_{1}\right], E\right) \\
v & \longmapsto(\Upsilon v)(t)=\int_{0}^{t} S_{\alpha}(t-s) v(s) d s .
\end{aligned}
$$

By virtue of Lemma 2.1, we know that $\Upsilon \circ S_{F}$ is a closed graph operator. Moreover, for every $t \in\left[0, t_{1}\right]$, we get

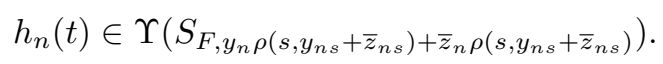

Since $z_{n} \rightarrow z_{*}$ and $h_{n} \rightarrow h_{*}$, it follows, that for every $t \in\left[0, t_{1}\right]$,

$$
h_{*}(t)=\int_{0}^{t} S_{\alpha}(t-s) v_{*}(s) d s,
$$

for some $v_{*} \in S_{F, y_{*} \rho\left(s, y_{* s}+\bar{z}_{* s}\right)+\bar{z}_{*} \rho\left(s, y_{* s}+\bar{z}_{* s}\right)}$.

Similarly, for any $t \in\left(t_{i}, t_{i+1}\right], i=1, \ldots, m$, we have

$$
\begin{aligned}
h_{n}(t) & =T_{\alpha}\left(t-t_{i}\right)\left[y_{n}\left(t_{i}^{-}\right)+\bar{z}_{n}\left(t_{i}^{-}\right)+I_{i}\left(y_{n}\left(t_{i}^{-}\right)+\bar{z}_{n}\left(t_{i}^{-}\right)\right)\right] \\
& +\int_{t_{i}}^{t} S_{\alpha}(t-s) v_{n}(s) d s .
\end{aligned}
$$

We must prove that there exists $v_{*} \in S_{F, y_{*} \rho\left(s, y_{* s}+\bar{z}_{* s}\right)+\bar{z}_{*} \rho\left(s, y_{* s}+\bar{z}_{* s}\right)}$ such that, for each $t \in\left(t_{i}, t_{i+1}\right]$,

$$
\begin{aligned}
h_{*}(t) & =T_{\alpha}\left(t-t_{i}\right)\left[y_{*}\left(t_{i}^{-}\right)+\bar{z}_{*}\left(t_{i}^{-}\right)+I_{i}\left(y_{*}\left(t_{i}^{-}\right)+\bar{z}_{*}\left(t_{i}^{-}\right)\right)\right] \\
& +\int_{t_{i}}^{t} S_{\alpha}(t-s) v_{*}(s) d s .
\end{aligned}
$$

Now, for every $t \in\left(t_{i}, t_{i+1}\right], i=1, \ldots, m$, we have

$$
\begin{aligned}
& \|\left(h_{n}(t)-T_{\alpha}\left(t-t_{i}\right)\left[y_{n}\left(t_{i}^{-}\right)+\bar{z}_{n}\left(t_{i}^{-}\right)+I_{i}\left(y_{n}\left(t_{i}^{-}\right)+\bar{z}_{n}\left(t_{i}^{-}\right)\right)\right]\right) \\
& \quad-\left(h_{*}(t)-T_{\alpha}\left(t-t_{i}\right)\left[y_{*}\left(t_{i}^{-}\right)+\bar{z}_{*}\left(t_{i}^{-}\right)+I_{i}\left(y_{*}\left(t_{i}^{-}\right)+\bar{z}_{*}\left(t_{i}^{-}\right)\right)\right]\right) \| \\
& \quad \rightarrow 0 \text { as } n \rightarrow \infty .
\end{aligned}
$$

Consider the linear continuous operator:

$$
\begin{aligned}
\Upsilon: L^{1}\left(\left(t_{i}, t_{i+1}\right], E\right) & \longrightarrow C\left(\left(t_{i}, t_{i+1}\right], E\right) \\
v & \longmapsto(\Upsilon v)(t)=\int_{t_{i}}^{t} S_{\alpha}(t-s) v(s) d s .
\end{aligned}
$$

From Lemma 2.1, it follows that $\Upsilon \circ S_{F}$ is a closed graph operator. Also, from the definition of $\Upsilon$, we have that, for every $t \in\left(t_{i}, t_{i+1}\right], i=1, \ldots, m$,

$$
\begin{aligned}
& \left(h_{n}(t)-T_{\alpha}\left(t-t_{i}\right)\left[y_{n}\left(t_{i}^{-}\right)+\bar{z}_{n}\left(t_{i}^{-}\right)+I_{i}\left(y_{n}\left(t_{i}^{-}\right)+\bar{z}_{n}\left(t_{i}^{-}\right)\right)\right]\right) \\
& \quad \in \Upsilon\left(S_{F, y_{n} \rho\left(s, y_{n s}+\bar{z}_{n s}\right)+\bar{z}_{n} \rho\left(s, y_{n s}+\bar{z}_{n s}\right)}\right) .
\end{aligned}
$$


Since $z_{n} \rightarrow z_{*}$, for some $v_{*} \in S_{F, y_{*} \rho\left(s, y_{* s}+\bar{z}_{* s}\right)+\bar{z}_{*} \rho\left(s, y_{* s}+\bar{z}_{* s}\right)}$ it follows that, for every $t \in\left(t_{i}, t_{i+1}\right]$, we have

$$
\begin{aligned}
h_{*}(t) & =T_{\alpha}\left(t-t_{i}\right)\left[y_{*}\left(t_{i}^{-}\right)+\bar{z}_{*}\left(t_{i}^{-}\right)+I_{i}\left(y_{*}\left(t_{i}^{-}\right)+\bar{z}_{*}\left(t_{i}^{-}\right)\right)\right] \\
& +\int_{t_{i}}^{t} S_{\alpha}(t-s) v_{*}(s) d s .
\end{aligned}
$$

Hence the multivalued operator $P$ is upper semi-continuous.

Step 6: A priori bounds.

Now it remains to show that the set

$$
\mathcal{E}=\left\{z \in \mathcal{B}_{2}: \lambda z \in P z, \text { for some } \lambda>1\right\}
$$

is bounded.

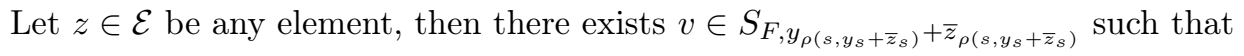

$$
z(t)=\lambda^{-1}\left\{\begin{array}{lr}
\int_{0}^{t} S_{\alpha}(t-s) v(s) d s, & t \in\left[0, t_{1}\right] \\
T_{\alpha}\left(t-t_{1}\right)\left[y\left(t_{1}^{-}\right)+\bar{z}\left(t_{1}^{-}\right)+I_{1}\left(y\left(t_{1}^{-}\right)+\bar{z}\left(t_{1}^{-}\right)\right)\right] & \\
+\int_{t_{1}}^{t} S_{\alpha}(t-s) v(s) d s, & t \in\left(t_{1}, t_{2}\right] \\
\quad \vdots & \\
T_{\alpha}\left(t-t_{m}\right)\left[y\left(t_{m}^{-}\right)+\bar{z}\left(t_{m}^{-}\right)+I_{m}\left(y\left(t_{m}^{-}\right)+\bar{z}\left(t_{m}^{-}\right)\right)\right] & \\
+\int_{t_{m}}^{t} S_{\alpha}(t-s) v(s) d s, & t \in\left(t_{m}, T\right] .
\end{array}\right.
$$

Then for each $t \in\left[0, t_{1}\right]$ we have,

$$
\begin{aligned}
\|z(t)\| & \leq \int_{0}^{t}\left\|S_{\alpha}(t-s)\right\|\|v(s)\| d s \\
& \leq \widetilde{M}_{s} \int_{0}^{t}(t-s)^{\alpha-1}\|v(s)\| d s
\end{aligned}
$$

for $t \in\left(t_{i}, t_{i+1}\right], i=1, \ldots, m$, we get

$$
\begin{aligned}
\|z(t)\| & \leq\left\|T_{\alpha}\left(t-t_{i}\right)\right\|\left(\left\|z\left(t_{i}^{-}\right)\right\|+\left\|I_{i}\left(z\left(t_{i}^{-}\right) \|\right)+\int_{t_{i}}^{t}\right\| S_{\alpha}(t-s)\|\| v(s) \| d s\right. \\
& \leq \widetilde{M}_{T}\left\|z\left(t_{i}^{-}\right)\right\|+\widetilde{M}_{T} \Omega+\widetilde{M}_{s} \int_{0}^{t}(t-s)^{\alpha-1}\|v(s)\| d s .
\end{aligned}
$$

Then for all $t \in[0, T]$, we have

$$
\begin{aligned}
\|z(t)\| & \leq \frac{\widetilde{M}_{T} \Omega}{1-\widetilde{M}_{T}}+\frac{\widetilde{M}_{s} T^{\alpha}}{\alpha\left(1-\widetilde{M}_{T}\right)} \int_{0}^{t} \mu(s) \psi\left(\left(C_{2}^{*}+L^{\phi}\right)\|\phi\|_{\mathcal{B}}+\left(C_{1}^{*}+1\right)\|z(s)\|\right) d s \\
& \leq \frac{\widetilde{M}_{T} \Omega}{1-\widetilde{M}_{T}}+\frac{\widetilde{M}_{s} T^{\alpha}}{\alpha\left(1-\widetilde{M}_{T}\right)} \int_{0}^{t} \mu(s) \psi\left(\beta_{1}+\beta_{2}\|z(t)\|\right) d s .
\end{aligned}
$$

Then

$$
\begin{aligned}
\beta_{1}+\beta_{2}\|z(t)\| & \leq \beta_{1}+\frac{\beta_{2} \widetilde{M}_{T} \Omega}{1-\widetilde{M}_{T}}+\frac{\beta_{2} \widetilde{M}_{s} T^{\alpha}}{\alpha\left(1-\widetilde{M}_{T}\right)} \int_{0}^{t} \mu(s) \psi\left(\beta_{1}+\beta_{2}\|z(s)\|\right) d s \\
& \leq \omega_{1}+\omega_{2} \int_{0}^{t}\left[\mu(s) \psi\left(\beta_{1}+\beta_{2}\|z(s)\|\right)\right] d s .
\end{aligned}
$$


Let

$$
m(t):=\sup \left\{\beta_{1}+\beta_{2}\|z(s)\|: 0 \leq s \leq t\right\}, t \in J .
$$

By the previous inequality, we have

$$
m(t) \leq \omega_{1}+\omega_{2} \int_{0}^{t}[\mu(s) \psi(m(s))] d s .
$$

Let us take the right-hand side of the above inequality as $v(t)$. Then we have

$$
m(t) \leq v(t) \text { for all } t \in J,
$$

with

$$
v(0)=\omega_{1},
$$

and

$$
v^{\prime}(t)=\omega_{2} \mu(t) \psi(m(t)), \quad \text { a.e. } t \in J .
$$

Using the nondecreasing character of $\psi$ we get

$$
v^{\prime}(t) \leq \omega_{2} \mu(t) \psi(v(t)), \quad \text { a.e. } t \in J .
$$

Integrating from 0 to $t$ we get

$$
\int_{0}^{t} \frac{v^{\prime}(s)}{\psi(v(s))} d s \leq \omega_{2} \int_{0}^{t} \mu(s) d s .
$$

By a change of variable we get

$$
\int_{v(0)}^{v(t)} \frac{d u}{\psi(u)} \leq \omega_{2} \int_{0}^{t} \mu(s) d s .
$$

Using the condition (4), this implies that for each $t \in J$, we have

$$
\int_{v(0)}^{v(t)} \frac{d u}{\psi(u)} \leq \omega_{2} \int_{0}^{t} \mu(s) d s \leq \omega_{2} \int_{0}^{T} \mu(s) d s<\int_{v(0)}^{+\infty} \frac{d u}{\psi(u)} .
$$

Thus, for every $t \in J$, there exists a constant $\Lambda$ such that $v(t) \leq \Lambda$ and hence $m(t) \leq \Lambda$. Since $\|z\|_{\mathcal{B}_{2}} \leq m(t)$, we have $\|z\|_{\mathcal{B}_{2}} \leq \Lambda$.

This shows that the set $\mathcal{E}$ is bounded. As a consequence of the Lemma 2.2, we deduce that the operator $P$ has a fixed point which gives rise to a mild solution of the problem (1).

\section{An ExAmple}

We consider the impulsive fractional integro-differential problem:

$$
\begin{aligned}
\frac{\partial_{t}^{q}}{\partial t^{q}} v(t, \zeta) & \in \frac{\partial^{2}}{\partial \zeta^{2}} v(t, \zeta)+\int_{-\infty}^{s} \eta(s, \tau-s, \zeta) G(\tau, v(\tau-\sigma(v(\tau, 0)), \zeta)) d \tau d s \\
v(t, 0) & =v(t, \pi)=0 \\
v(t, \zeta) & =v_{0}(\theta, \zeta), \quad-\infty<\theta \leq 0 \\
\Delta v\left(t_{k}\right)(\zeta) & =\int_{-\infty}^{t_{k}} p_{k}\left(t_{k}-y\right) d y \cos \left(v\left(t_{k}\right)(\zeta)\right)
\end{aligned}
$$

where $0<q<1, t \in[0, T], \sigma \in C(\mathbb{R},[0, T]), \zeta \in[0, \pi], p_{k}: \mathbb{R} \rightarrow \mathbb{R}, k=1,2, \ldots, m$, and $G:[0, T] \times \mathcal{B} \rightarrow P(\mathbb{R})$ is an u.s.c. multivalued map with compact convex values. 
Set $E=L^{2}([0, \pi])$ and define $A$ by

$$
\begin{gathered}
D(A)=\left\{u \in E: u^{\prime \prime} \in E, u(0)=u(\pi)=0\right\}, \\
A u=u^{\prime \prime} .
\end{gathered}
$$

It is well known that $A$ is the infinitesimal generator of an analytic semigroup $(S(t))_{t \geq 0}$ on $E$. For the phase space, we choose $\mathcal{B}=\mathcal{B}_{\gamma}$ defined by

$$
\mathcal{B}_{\gamma}:=\left\{\phi \in C((-\infty, 0], E): \lim _{\theta \rightarrow-\infty} e^{\gamma \theta} \phi(\theta) \text { exists in } E\right\}
$$

endowed with the norm

$$
\|\phi\|=\sup \left\{e^{\gamma \theta}|\phi(\theta)|: \theta \leq 0\right\} .
$$

Notice that the phase space $\mathcal{B}_{\gamma}$ satisfies axioms (A1) - (A3).

For $t \in[0, T], \zeta \in[0, \pi]$ and $\varphi \in \mathcal{B}_{\gamma}$ we set

$$
\begin{aligned}
& x(t)(\zeta)=v(t, \zeta) \\
& \phi(\theta)(\zeta)=v_{0}(\theta, \zeta) \\
& F(t, \varphi, x(t))(\zeta)=\int_{-\infty}^{0} \eta(t, \theta, \zeta) G(t, \varphi(0, \zeta)) d \theta \\
& \rho(t, \varphi)=t-\sigma(\varphi(0,0)) \\
& I_{k}\left(x\left(t_{k}^{-}\right)\right)(\zeta)=\int_{-\infty}^{0} p_{k}\left(t_{k}-y\right) d y \cos \left(x\left(t_{k}\right)(\zeta)\right), \quad k=1,2, \ldots, m .
\end{aligned}
$$

We can show that problem (5) is an abstract formulation of problem (1). The following result is a direct consequence of Theorem 3.2.

Proposition 4.1. Let $\varphi \in \mathcal{B}$ be such that $\left(H_{\varphi}\right)$ holds, and let $t \rightarrow \varphi_{t}$ be continuous on $\mathcal{R}\left(\rho^{-}\right)$. Then there exists a mild solution of $(5)$.

\section{REFERENCES}

[1] S. Abbas, M. Benchohra, G.M. N'Guérékata, Topics in Fractional Differential Equations, Springer, New York, 2012.

[2] S. Abbas, M. Benchohra, G.M. N'Guérékata, Advanced Fractional Differential and Integral Equations, Nova Science Publishers, New York, 2015.

[3] R. P. Agarwal, B. De Andrade, G. Siracusa, On fractional integro-difierential equations with state-dependent delay, Computers \& Mathematics with Applications, 62 (2011), 1143-1149.

[4] W. G. Aiello, H. I. Freedman, J. Wu, Analysis of a model representing stagestructured population growth with state-dependent time delay, SIAM Journal on Applied Mathematics, 52 (3) (1992), 855-869.

[5] K. Aissani, M. Benchohra, Existence results for fractional integro-differential equations with state-dependent delay, Advances in Dynamical Systems and Applications, 9 (1) (2014), 17-30.

[6] K. Aissani, M. Benchohra, K. Ezzinbi, Fractional integro-differential inclusions with state-dependent delay, Discussiones Mathematicae Differential Inclusions, Control and Optimization, 34 (2) (2014), 153-167. 
[7] K. Aissani, M. Benchohra, M. Meghnafi, Controllability of Impulsive fractional evolution equations with state-dependent delay, Memoirs on Differential equations and Mathematical Physics, 73 (2018), 1-20.

[8] D. Araya, C. Lizama, Almost automorphic mild solutions to fractional differential equations, Nonlinear Analysis, 69 (11) (2008), 3692-3705.

[9] D. D. Bainov, P. S. Simeonov, Systems with Impulse Effect, Ellis Horwood Ltd., Chichister, 1989.

[10] V. Barbu, Nonlinear Semigroups and Differential Equations in Banach Spaces, Noordhoff International Publishing, Leiden, 1976.

[11] E. Bazhiekova. Fractional Evolution Equations in Banach Spaces, Ph.D. Thesis, Eindhoven University of Technology, 2001.

[12] M. Benchohra, B.A. Slimani, Existence and uniqueness of solutions to impulsive fractional differential equations, Electronic Journal of Differential Equations, 2009 (10) (2009), 1-11.

[13] Y. Cao, J. Fan, T. C. Gard, The effects of state-dependent time delay on a stagestructured population growth model, Nonlinear Analysis, 19 (2) (1992), 95-105.

[14] A. Cernea, On a fractional differential inclusion with boundary condition, Studia Universitatis Babeş-Bolyai Mathematica, LV (2010), 105-113.

[15] F. Chen, D. Sun, J. Shi, Periodicity in a food-limited population model with toxicants and state dependent delays, Journal of Mathematical Analysis and Applications, 288 (1) (2003), 136-146.

[16] L. Debnath, D. Bhatta, Integral Transforms and Their Applications(Second Edition), CRC Press, 2007.

[17] A. Domoshnitsky, M. Drakhlin, E. Litsyn, On equations with delay depending on solution, Nonlinear Analysis, 49 (5) (2002), 689-701.

[18] K. Deimling, Multivalued Differential Equations, Walter De Gruyter, Berlin-New York, 1992.

[19] A.M.A. El-Sayed, A.G. Ibrahim. Multivalued fractional differential equations of arbitrary orders, Applied Mathematics and Computation, 68 (1995), 15-25.

[20] L. Górniewicz, Topological Fixed Point Theory of Multivalued Mappings, Mathematics and its Applications, 495, Kluwer Academic Publishers, Dordrecht, 1999.

[21] J. K. Hale, J. Kato, Phase space for retarded equations with infinite delay, Funkcialaj Ekvacioj, 21 (1978), 11-41.

[22] F. Hartung, Linearized stability in periodic functional differential equations with state-dependent delays, Journal of Computational and Applied Mathematics, 174 (2) (2005), 201-211.

[23] F. Hartung, Parameter estimation by quasilinearization in functional differential equations with state-dependent delays: a numerical study, Nonlinear Analysis, 47 (7) (2001), 4557-4566.

[24] F. Hartung, T. L. Herdman, J. Turi, Parameter identification in classes of neutral differential equations with state-dependent delays, Nonlinear Analysis, 39 (3) (2000), 305-325. 
[25] F. Hartung, J. Turi, Identification of parameters in delay equations with statedependent delays, Nonlinear Analysis, 29 (11) (1997), 1303-1318.

[26] E. Hernández, M. A. Mckibben, On state-dependent delay partial neutral functionaldifferential equations, Applied Mathematics and Computation, 186 (1) (2007), 294301.

[27] E. Hernández, M. A. Mckibben, H. R. Henriquez, Existence results for partial neutral functional differential equations with state-dependent delay, Mathematical and Computer Modelling, 49 (2009), 1260-1267.

[28] E. Hernández, A. Prokopczyk, L. Ladeira, A note on partial functional differential equations with state-dependent delay, Nonlinear Analysis: Real World Applications, 7 (2006), 510-519.

[29] Y. Hino, S. Murakami, T. Naito, Functional Differential Equations with Unbounded Delay, Springer-Verlag, Berlin, 1991.

[30] Sh. Hu, N. Papageorgiou, Handbook of Multivalued Analysis, Volume I: Theory, Kluwer Academic Publishers, Dordrecht, Boston, London, 1997.

[31] A. A. Kilbas, Hari M. Srivastava, Juan J. Trujillo, Theory and Applications of Fractional Differential Equations, Elsevier Science B.V., Amsterdam, 2006.

[32] Y. Kuang, H. L. Smith, Slowly oscillating periodic solutions of autonomous statedependent delay equations, Nonlinear Analysis, 19 (9) (1992), 855-872.

[33] V. Lakshmikantham, D.D. Bainov, P.S. Simeonov, Theory of Impulsive Differential Equations, World Scientific, NJ, 1989.

[34] V. Lakshmikantham, S. Leela, J. Vasundhara, Theory of Fractional Dynamic Systems, Cambridge Academic Publishers, Cambridge, 2009.

[35] A. Lasota, Z. Opial, An application of the Kakutani-Ky Fan theorem in the theory of ordinary differential equations, Bulletin L'Académie Polonaise des Science, Série des Sciences Mathématiques, Astronomiques et Physiques (BAPMAM), 13 (1965), $781-786$.

[36] C. Lizama, Regularized solutions for abstract Volterra equations, Journal of Mathematical Analysis and Applications, 243 (2000), 278-292.

[37] T. Maraaba, D. Baleanu, F. Jarad, Existence and uniqueness theorem for a class of delay differential equations with left and right Caputo fractional derivatives, Journal of Mathematical Physics, 49 (8) (2008), Article ID 083507, 11 pages.

[38] T. Maraaba, F. Jarad, D. Baleanu, On the existence and the uniqueness theorem for fractional differential equations with bounded delay within Caputo derivatives, Science in China Series A - Mathematics, 51 (10) (2008), 1775-1786.

[39] M. Martelli, A Rothe's type theorem for non-compact acyclic-valued maps, Bollettino dell'Unione Matematica Italiana, 11 (3) (1975), 70-76.

[40] M. Meghnafi, K. Aissani, M. Benchohra, Impulsive fractional evolution equations with state-dependent delay, Nonlinear Studies, 22 (4) (2015), 659-671.

[41] K. S. Miller, B. Ross, An Introduction to the Fractional Calculus and Differential Equations, John Wiley, New York, 1993.

[42] I. Podlubny, Fractional Differential Equations, Acadmic press, New York, 1993. 
[43] J. Prüss, Evolutionary Integral Equations and Applications, Monographs in Mathematics, Birkhäuser, Basel, Switzerland, 1993.

[44] A. V. Rezounenko, Partial differential equations with discrete and distributed statedependent delays, Journal of Mathematical Analysis and Applications, 326 (2) (2007), 1031-1045.

[45] X.B. Shu, Y.Z. Lai, Y. Chen. The existence of mild solutions for impulsive fractional partial differential equations, Nonlinear Analysis, 74 (2011), 2003-2011.

[46] R. Torrejón, Positive almost periodic solutions of a state-dependent delay nonlinear integral equation, Nonlinear Analysis, 20 (12) (1993), 1383-1416.

[47] Z. Yang, J. Cao, Existence of periodic solutions in neutral state-dependent delays equations and models, Journal of Computational and Applied Mathematics, 174 (1) (2005), 179-199.

[48] Y. Zhou, Fractional Evolution Equations and Inclusions: Analysis and Control, Elsevier / Academic Press, London, 2016.

\section{Khalida Aissani}

UNIVERSITY OF BECHAR

P.O. Box 417, 08000, BECHAR

Algeria

E-mail address: aissani_k@yahoo.fr

\section{MoufFaK Benchohra}

LABORATORY OF MATHEMATICS

Djillali Liabes University of Sidi Bel-Abbès

P.O. Box 89

22000 Sidi BEL-ABBÈs

Algeria

Department of Mathematics

College of Science

King Saud University

P.O. Box 2455

RIYADH 11451

SAudi Arabia

E-mail address: benchohra@yahoo.com 